\title{
Meduloblastoma bilateral: reporte de caso y revisión de la literatura
}

\author{
Alfonso Pacheco-Hernández ${ }^{4}$, Leonardo Domínguez de la Ossa ${ }^{7}$, Ginna De la Rosa ${ }^{4}$, Cristian Camilo Blanco-Teherán ${ }^{1,2}$, \\ Romario Mendoza-Flórez ${ }^{1,2}$, Yancarlos Ramos-Villegas ${ }^{1,2}$, Huber S. Padilla-Zambrano ${ }^{1,2,3}$, Loraine Quintana-Pájaro ${ }^{1,2}$, \\ Hugo Corrales-Santander ${ }^{5,6}$, Alexis Narvaez-Rojas ${ }^{3}$, Luis Rafael Moscote-Salazar ${ }^{2,3,4}$ \\ 1 Estudiante de Medicina, Universidad de Cartagena, Cartagena de Indias, Colombia. \\ 2 Centro de Investigaciones Biomédicas (CIB), Facultad de Medicina, Universidad de Cartagena, Cartagena Colombia. \\ 3 RED LATINO Organización Latinoamericana de Trauma y cuidado Neurointensivo. \\ 4 Médico, Especialista en Neurocirugía, Faculta de Medicina, Universidad de Cartagena, Cartagena de Indias, Bolívar. \\ 5 Médico, Magíster en Toxicología, Coordinador Centro de Investigaciones Biomédicas (CIB), Facultad de Medicina, Univer- \\ sidad de Cartagena, Cartagena Colombia. \\ 6 Programa de Medicina, Corporación Universitaria Rafael Núñez, Cartagena, Colombia. \\ 7 Neurocirujano Pediatrico, Universidad de Cartagena, Cartagena de Indias, Colombia.
}

Rev. Chil. Neurocirugía 44: 175-180, 2018

\begin{abstract}
Resumen
Uno de los tumores más frecuentes en la edad pediátrica es el meduloblastoma; esta neoplasia causa al menos $30 \%$ de los tumores en la edad pediátrica. Las manifestaciones clínicas dependerán de la ubicación de la lesión. La aparición del meduloblastoma bilateral es un fenómeno rara vez descrito en la literatura. Presentamos un caso de esta interesante patología.
\end{abstract}

Palabras clave: Meduloblastoma bilateral, tumor cerebral, pediatría.

\begin{abstract}
One of the most frequent tumors in pediatric age is medulloblastoma; this neoplasm causes at least $30 \%$ of tumors in the pediatric age. Clinical manifestations will depend on the location of the lesion. The appearance of bilateral medulloblastoma is a phenomenon rarely described in the literature. We present a case study of this pathology.
\end{abstract}

Key words: Bilateral medulloblastoma, brain tumor, pediatrics.

\section{Introducción}

El meduloblastoma es el tumor cerebral maligno de tipo embrionario más frecuente en la etapa de la infancia, representando entre el 15 y $30 \%$ de éstos y se presenta en los adultos con menor incidencia. Son frecuentes en el cerebelo y el IV ventrículo, sin embargo, el meduloblastoma bilateral cerebeloso es poco frecuente. La Organización Mundial de la Salud (OMS), clasificó los meduloblastomas dentro del grupo de los tumores de grado IV por sus características malignas, actividad mitótica elevada y rápida evolución ${ }^{1-4}$. Esta neoplasia era conocida como "glioma cerebeloso" y en el año 1925 Bailey y Cushing utilizaron el término de "meduloblastoma"5. Estos tumores pueden ocasionarse por diversas mutaciones en el material genético y se relaciona con diversos síndromes. Las manifestaciones clínicas características son el aumento de la presión intracraneal, acompañada de hidrocefalia ${ }^{3,5,6}$. La re- sección quirúrgica es el tratamiento de primera línea y su pronóstico depende de la edad del paciente, siendo mejor en lactantes ${ }^{1,4}$. Presentamos el caso de un meduloblastoma bilateral en niños y una revisión de la literatura.

\section{Descripción del caso}

Femenina de 6 años de evolución con cuadro clínico de cefalea holocraneana de 1 año de evolución que progreso 
con vómitos de contenido alimenticio. Como antecedente relevante hermano con tumor del SNC (No recuerdan estirpe). Es llevado por padres a Hospital pediátrico de Cartagena de Indias, donde es evaluado, se evidencia paciente Consciente, pupilas isocóricas reactivas, papiledema bilateral, simetría facial, fuerza muscular y sensibilidad conservada, marcha atáxica, dismetría bilateral.

Se realiza TAC cerebral que muestra lesiones hipodensas en cerebelo bilateralmente. (Figura 1 a y b). Se realiza RMN de cerebro que revela lesiones intraparenquimatosas. Se decide realizar craneotomía suboccipital bilateral y resección total de lesiones intraxiales. El reporte de histopatología revela una "célula en forma de zanahoria" con núcleos hipercromáticos polimórficos y de aspecto de "Rosetas de Homer Wright", lo cual es compatible con las características histopatologicas de los meduloblastomas. Paciente evoluciona de manera satisfactoria en su posoperatorio. La paciente es enviada a domicilio a los 10 días. Se realiza RMN de cerebro con gadolinio que descarta recidiva tumoral (Figura 2 a, b y c). Actualmente continua en manejo por neurocirugía pediátrica y oncología. La Figura 3 se muestran los hallazgos histológicos.

\section{Discusión}

El meduloblastoma es un tumor cerebral de tipo embrionario, carácter maligno y de aspecto sólido, catalogado como tumor neuroectodérmico primitivo, el cual tiene su origen en la fosa posterior del cráneo y afecta principalmente al cerebelo y al IV ventrículo; además, en este tipo de tumores se destaca la expresión de los marcadores de las células precursoras del Sistema Nervioso Central ${ }^{1,3,7-9}$. Según la Organización Mundial de la Salud - OMS, los meduloblastomas se clasifican dentro del grupo de los tumores de grado IV debido que son lesionas muy malignas, con gran actividad mitótica, evolución rápida y tendencia a producir áreas de necrosis ${ }^{4,9}$. En un principio esta afección fue conocida como glioma cerebeloso y no fue hasta el año 1925 cuando Bailey y Cushing lo denominaron como se conoce actualmente ${ }^{5}$.

\section{Clasificación histológica}

Los meduloblastomas son tumores que

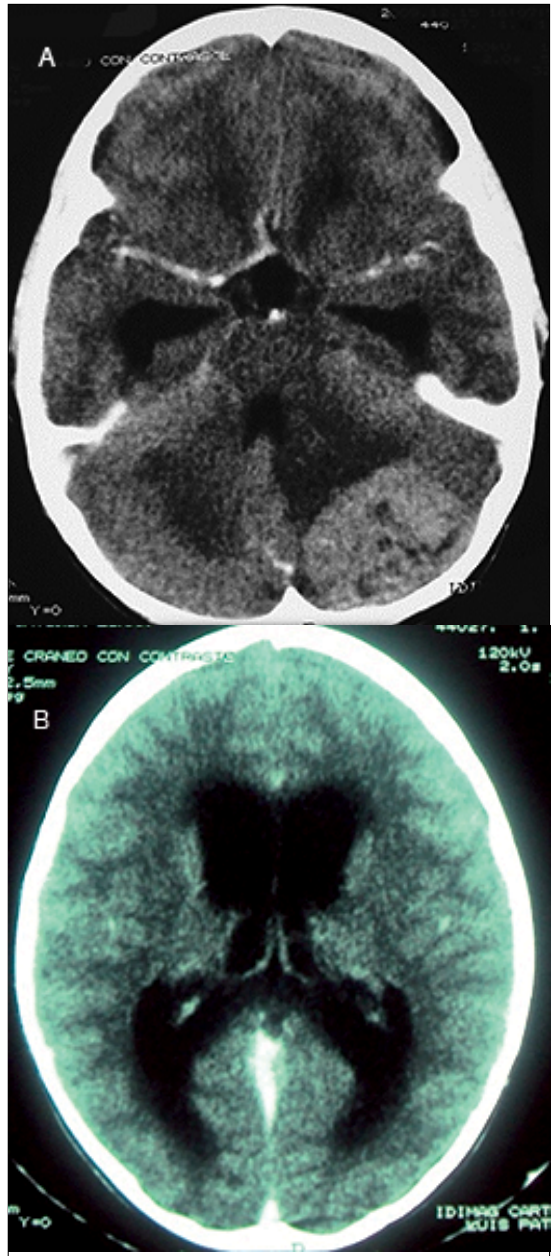

Figura 1 a y b. TAC cerebral que evidencia lesiones en cerebro bilateral con ventriculomegalia.

dividen teniendo en cuenta las características histopatológicas: 1) Meduloblastoma clásico; 2) Meduloblastoma desmoplásico/nodular; 3) Meduloblastoma con nodularidad extensa; 4) Meduloblastoma anaplásico; y 5) Meduloblastoma de células grandes ${ }^{4,5,7,10}$. Por su histología y patrón de sus células son muy semejantes, los meduloblastomas de tipo desmoplásico/nodular y con nodularidad extensa se clasifican un solo grupo al cual se denominan meduloblastomas desmoplásicos. Por otro lado, la clasificación anaplásica y de células grandes en ocasiones tienden a aparecer juntos, por esa razón se denominan meduloblastomas de células grandes/anaplásico ${ }^{10}$

El meduloblastoma clásico es el tipo más frecuente, representando entre el 70 y $80 \%$ de todos los casos presentados. Histológicamente, se caracte-

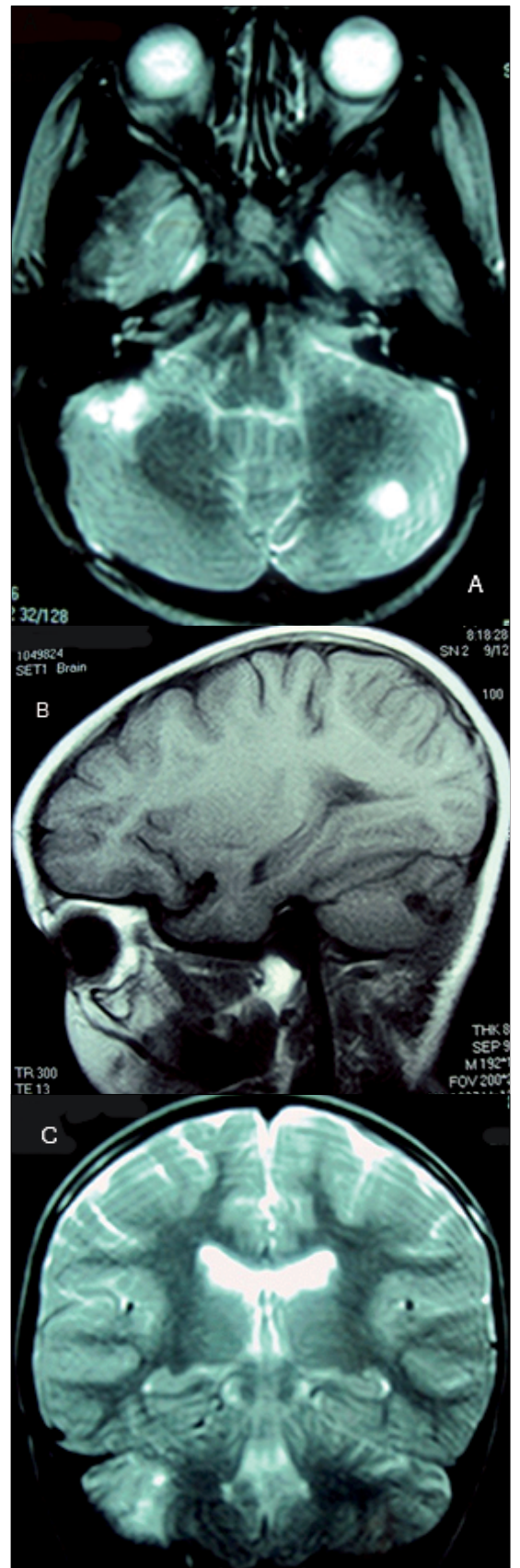

Figura 2 a, b y c. RMN de cerebro con gadolinio de control a los 4 meses que descarta recidiva tumoral.

rizan por presentar pleomorfismo en las células, las cuales son de pequeños tamaños, redondos u ovalados, y se organizan en hojas. En ocasiones existen rosetas de Homer-Wright. Además, las alteraciones en las en la vía de señalización Wnt y Shh son frecuentes. Son poco frecuentes en niños menores a 3 años, sin embargo, 
presentan una incidencia mayor a los 10 y 20 años. Se ha sugerido que estos tumores son originados de células progenitoras de la matriz del ventrículo, fundamentándose en el análisis de la expresión de Calbindin-D28K. Los meduloblastomas clásicos aparecen predominantemente en el vermis cerebeloso. Además, tienden a diseminarse por el líquido cefalorraquídeo hasta llegar al canal espinal. En ocasiones evolucionan a un meduloblastoma anaplásico ${ }^{6,10,11 .}$.

La variante desmoplásica, que incluye los tumores desmoplásico/nodular, y con nodularidad extensa, se observa con mayor frecuencia en los pacientes adultos que en los niños, representando entre el 30 y $40 \%$ de todos los meduloblastomas adultos, y entre el 10 y $20 \%$ de estos tumores en infantiles ${ }^{6,11}$. Esta línea de tumores se caracteriza por presentar nódulos, compuestos por células neurocíticas diferenciadas, los cuales se encuentran dispersos entre células indiferenciadas y zonas de desmoplasia. Singularmente, el meduloblastoma con nodularidad extensa tiene un alto número de nódulos irregulares mientras que los meduloblastomas demoplásico/nodular presentan nódulos escasos pero diseminados por zonas de tejido conectivo, ricas en fibras de colágeno. Todos estos tumores han presentado defectos en la vía de señalización Sonic Hedgehog $(\mathrm{Shh})^{9,10}$. Debido que se ha analizado la expresión del receptor del factor de crecimiento nervioso de baja afinidad (P75NTR o NGFR) y MATH1 se ha sugerido que estos tumores se originan de las células de la capa granular externa del cerebelo ${ }^{11}$.

Los meduloblastomas desmoplásicos, a diferencia de la variante clásica, se encuentran predominantemente en los hemisferios cerebelosos. En los pacientes pediátricos, las metástasis fuera del Sistema Nervioso Central son muy extrañas, pero el líquido cefalorraquídeo si puede encontrarse afectado. En los adultos, el $50 \%$ de los casos se localizan en la fosa posterior del cráneo, el $5 \%$ presentan metástasis por fuera del Sistema Nervioso Central, con frecuencia presentándose en los huesos y en el $30 \%$ de los pacientes, el líquido cefalorraquídeo se encuentra afectado ${ }^{6,11}$.

El subgrupo de los meduloblastomas de células grandes/anaplásico representa entre en 4 y $20 \%$ de todos es-

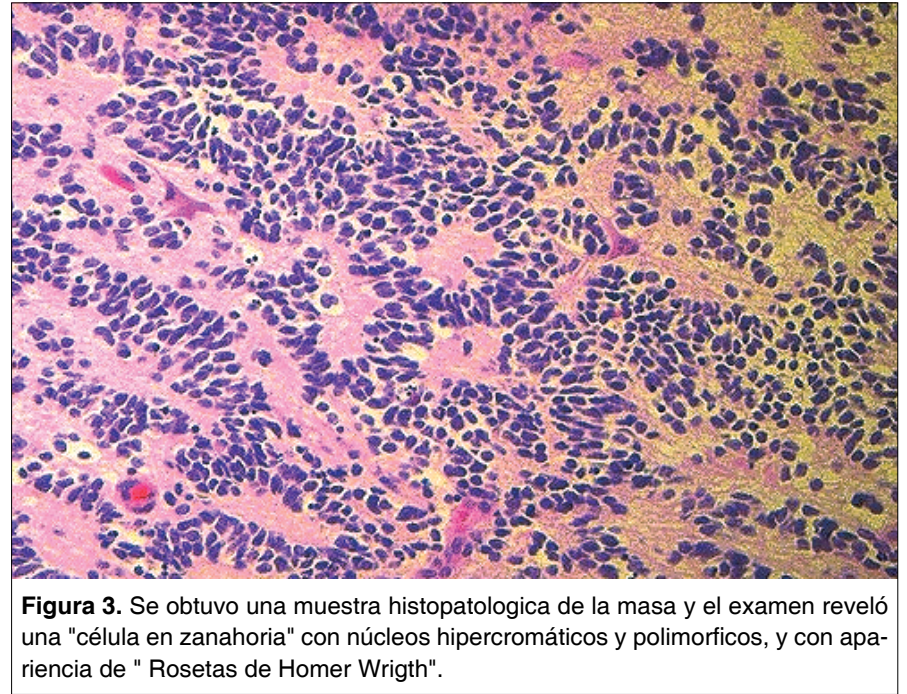

tos tumores, tienen un comportamiento muy agresivo y un mal pronóstico debido a su capacidad de hacer metástasis a través del fluido cerebroespinal y llegar por fuera del Sistema Nervioso Central ${ }^{5,10}$. Según su histología, los meduloblastomas de células grandes, como su nombre lo indica, presentan células de un tamaño aumentado, las cuales son redondas y con núcleos inmensos y nucléolos muy prominentes; además, tienen muy mal pronóstico. Mientras que tipo anaplásico posee células mal diferenciadas con pleomorfismos en sus núcleos, los cuales son angulares; este grupo tienen un mayor grado de atipia celular que los demás ${ }^{5,10}$

\section{Epidemiología}

El meduloblastoma es el tumor cerebral más frecuente en la infancia, representando entre el $15-30 \%$ de todos éstos. Además, constituye entre el $4-8 \%$ de los tumores intracraneales primitivos originados del neuroepitelio $\mathrm{y}$, aproximadamente, el $40 \%$ de los tumores encontrados en la fosa posterior del cráneo en pacientes pediátricos ${ }^{1-3,8,11-13}$. El mayor pico de incidencia es entre los 3 y 9 años, diagnosticándose en promedio a la edad de 5 años ${ }^{2,8,12}$. No obstante, esta condición puede ocurrir en la etapa adulta con una incidencia de 0,005 casos de 100.000 habitantes por año, representando así menos del $3 \%$ de los tumores del Sistema Nervioso en la adultez; con un pico mayor de incidencia entre los 30 y 40 años de edad ${ }^{2,5,6,8}$. Este tumor afecta más a los varones con una razón de 3:2 con respecto a las mujeres ${ }^{2,5}$. Los meduloblastomas bilaterales encontrados en los dos hemisferios del cerebelo son extremadamente raros. Además, la ubicación de la masa tumoral en la fosa posterior está relacionada con la edad; en los infantes tienen un predominio por el vermis y en los adultos se han encontrado con más frecuencia en el cerebelo ${ }^{3}$.

\section{Etiología}

Dentro de los principales factores etiológicos de esta neoplasia encontramos las deleciones encontradas en el brazo corto del cromosoma 17, la cual es la alteración más frecuente en el meduloblastoma representando entre el 40 y $50 \%$ de los tumores primarios; teniendo en cuenta las características histopatológicas del tumor, esta anomalía genética ocurre con mayor incidencia en el meduloblastoma clásico que en la clasificación desmoplásica. Otro factor causante de meduloblastoma puede ser la sobreexpresión y amplificación de los genes MYC o MYCN, el cual se presenta con una incidencia entre 5 a $10 \%$ del total de casos; teniendo en cuenta lo anterior, la reducción de los niveles de expresión de estos genes podría tener como consecuencia resultados muy óptimos ${ }^{6,10}$. Además, esta enfermedad ha sido asociada a diversos síndromes, en los cuales se destacan el de Li-Fraumeni, el síndrome de Turcot, síndrome de Gorlin-Gotz, neurofibromatosis tipos 1 y 2 , entre otros $^{6,10}$. 


\section{Genética molecular}

La patogenia y alteraciones moleculares de estos tumores han sido pobremente entendidas. No obstante, varios autores han dividido a los meduloblastomas en cuatro variantes moleculares: 1) Subgrupo Wnt; 2) subgrupo Sonic hedgehog (Shh); 3) grupo 3 y 4) grupo $4^{7,9,10}$

La variante Wnt va estar conformada predominantemente por meduloblastomas clásicos, pero también se pueden encontrar tumores de células grandes/ anaplásicos que tengan un mejor pronóstico. En todos los casos de esta clasificación se presenta monosomía del cromosoma 6 . Además, como el producto del gen APC es un inhibidor de la vía Wnt, el principal mecanismo que hace que ocurra esta enfermedad es una mutación en la línea germinal de este gen. También, se van a presentar defectos en el gen CTNNB1, el cual codifica la $\beta$-catenina, ocasionando así alteraciones en esta vía de señalización. En general, los meduloblastomas Wnt pueden ocurrir a cualquier edad, pero con menos frecuencia en niños menores de 3 años, afectado en igual proporción a ambos sexos; además, la sobrevida de estos pacientes a largo plazo es mayor a $90 \%$, aproximadamente ${ }^{10}$.

El grupo de los meduloblastomas Sonic Hedgehog debe su nombre a la vía de señalización Sonic Hedgehog (Shh), la cual tiene como función el desarrollo de tejidos y órganos en los vertebrados. La activación de esta vía de señalización se ocasiona principalmente por mutaciones en la línea germinal de PTCH1, que es una característica en los pacientes con síndrome de Gorlin. Esta variante se presenta con más frecuencia en los tumores desmoplásicos y ocurre con mayor incidencia en los niños de 0 a 3 años y en las personas mayores a 16 años, con igual porcentaje de casos en ambos sexos ${ }^{10}$

Hasta el momento, las dos últimas clasificaciones moleculares, el grupo 3 y grupo 4, no han estado directamente relacionadas con alguna vía de señalización en particular como los otros subgrupos. Pero existen algunas características importantes de estas variantes. Por ejemplo, para el grupo 3 existe una amplificación del gen MYC ocasionando altos niveles en su expresión; además, los tumores de este grupo están conformados especialmente por meduloblastomas clásicos, pero la inciden- cia de los tumores de células grandes/ anaplásicos es mayor que en cualquier otra clasificación molecular. Por otra parte, aunque la patogenia de los tumores del grupo 4 no esté totalmente entendida, se ha detectado que en estos dos grupos existe una amplificación y sobreexpresión de un factor de transcripción participante en el desarrollo del cerebro, el gen OTX2; al igual que la existencia de isocromosoma $17 q$ en este par de grupos. La pérdida del cromosoma $X$ es una característica especial de los tumores del grupo 4 aunque no se encuentre en todos los casos. En general, los tumores de estos dos grupos pueden ocurrir con mayor frecuencia hasta los 16 años, viéndose rara vez en las personas adultas y con mayor proporción en el sexo masculino. Además, las metástasis son bastante frecuentes teniendo como consecuencia un mal pronóstico ${ }^{10}$.

\section{Fisiopatología}

Hasta el momento sigue no se ha ilustrado el origen de esta neoplasia. Sin embargo, existen diferentes teorías que proponen la histogénesis de estos. Por un lado, se explica su origen a partir de células germinales encontradas en el techo neuroepitelial del IV ventrículo, específicamente en el velo medular posterior, las cuales proliferan hacia arriba y en forma lateral. Por otro lado, una teoría intenta explicar el origen de la neoplasia a partir de las células de la capa granular externa, las cuales generan el desarrollo del cerebelo

\section{Manifestaciones clínicas}

Los signos y síntomas clínicos de estos pacientes dependen de la edad de presentación y la estatificación del mismo, es decir, este focalizado o diseminado. En los bebés y niños menores, pueden presentarse dolores de cabeza náuseas, vómitos, diplopía, nistagmo, papiledema; además, estos pacientes tienden a estar irritables a cualquier estímulo y existe un crecimiento del perímetro cefálico. Todo esto en consecuencia a la hipertensión intracraneal existente, relacionada con la hidrocefalia producida por la obstrucción que ocasiona la masa tumoral ${ }^{2,5,10}$. También puede aparecer ataxia troncal o hipotonía, como resultado de las alteraciones cerebelosas y la ubicación del tumor en el vermis ${ }^{5}$. En los niños mayores y en los pacientes adultos es más frecuente que se presenten masas laterales acompañadas con disdiadococinesia y ataxia de extremidad. En este grupo de pacientes, las manifestaciones pueden empezar con dolores de cabeza que se relacionan con la posición corporal y vómitos. El síndrome de Parinaud y el daño en la mirada vertical puede ser consecuencia de la presión ejercida al tronco encefálico dorsal ocasionada por la hidrocefalia. Además, es común que exista una parálisis en el Nervio Abducens generada cuando se compromete el núcleo del VI par craneal en el margen anterior del IV ventrículo ${ }^{5}$. En el caso de que la enfermedad esté en una etapa metastásica, las manifestaciones van a depender del lugar donde haya ocurrido la diseminación. Cuando la metástasis compromete el canal espinal, ocurrirá una compresión de la médula, y en el caso de que exista una metástasis hemisférica el paciente presentará convulsiones ${ }^{5}$.

\section{Diagnóstico}

Los meduloblastomas por lo general son diagnosticados en las dos primeras décadas de la vida, si el paciente presenta un examen neurológico alterado o si el medico sospecha de alguna patología intracraneal debido a la historia clínica del mismo, entonces son necesarias más pruebas diagnósticas. El estándar de oro para el diagnóstico de meduloblastoma es netamente imagenológico (TAC o la resonancia magnética) en donde se podrá observar una masa en fosa posterior, en algunos casos acompañada de hidrocefalia obstructiva. La resonancia magnética simple y contrastada es la mejor opción para diagnóstico del meduloblastoma debido a que permite observar la relación entre el tumor y las estructuras cerebrales vecinas, además proporciona con mayor claridad la diseminación del tumor. La punción lumbar utilizada para evaluar las células tumorales no está indicada preoperatoriamente debido a que podría causar complicaciones como hernia cerebral, esta se realiza alrededor de 20 días luego de la cirugía. En la actualidad no existen marcadores específicos que puedan considerarse estándar de oro para el diagnóstico de cada uno de los tipos de meduloblastoma (meduloblastoma clásico, meduloblastoma desmoplásico, meduloblatoma con modularidad extensa y meduloblastoma de células grandes y anaplásico) ${ }^{10,11,14}$.

El diagnóstico de la diseminación de 
los meduloblastomas se realiza a través de diferentes estudios y pruebas de laboratorio dentro de las que incluimos:

- Resonancia magnética de médula espinal preoperatoria o varias semanas después de la cirugía.

- Análisis de citológico de líquido cefalorraquídeo, por lo menos, entre 15 y 20 días luego de la cirugía para evitar falsos positivos.

Las imágenes en las que encontramos presentes extensión extramedular intradural, por lo general están asociadas a nódulos subaracnoideos asilados o a metástasis en gota en la región caudal del conducto espinal ${ }^{5}$.

\section{Imagenología}

El meduloblastoma tiene su origen en la línea media del vermis, localización más frecuente y pueden crecer en el IV ventrículo; es un tumor que radiológicamente en TAC se describe como una masa media lineal bien definida, de carácter hiperdenso debido a su enorme celularidad y homogéneo, es posible la presencia de calcio y la presencia del edema peritumoral, es bastante variada. En la primera TAC se pueden encontrar complicaciones tumorales dentro de las que se incluyen el grado de hidrocefalia secundario a la obstrucción e incluso signos de hipertensión intracraneal. En la resonancia magnética con frecuencia se observa un tumor de fosa posterior bien definido, característico por presentar baja señal en T1 y una señal alta en imágenes en la secuencia T2. La heterogeneidad en las secuencias T2 de la resonancia magnética acompañada de un patrón de "nido de abeja" se asocia con el meduloblastoma anaplásico, de igual manera se ha descrito un patrón "similar a uvas", asociado a meduloblastoma de extensa nodularidad. Es importante no considerar las características atípicas tales como las calcificaciones, regiones quísticas o necróticas, márgenes mal definidos como un estándar imprescindible a la hora de realizar el diagnóstico definitivo. En la espectroscopia de resonancia magnética se evalúan niveles aumentados de colina, muy por encima de las elevaciones respectivas presentadas en otras masas con ubicación en la fosa posterior, este hallazgo es sugestivo de rotación de las células tumorales. Dentro de las alteraciones también encontramos una disminución significativa en los niveles de $\mathrm{N}$-acetil aspartato (NAA), razón por la cual hay una relación de colina/NAA elevada. En cuanto a los meduloblastomas es importante tener en cuenta los niveles de taurina debido a la asociación que existe entre esta y la malignidad de dicho tumor debido su relación con la proliferación celular y la perdida de diferenciación de las células tumorales ${ }^{2,5}$.

\section{Diagnósticos diferenciales}

En los pacientes con fuerte sospecha de meduloblastoma es importante realizar diagnostico diferencial con entidades tales como tumor teratoide rabdoide atípico, carcinoma de plexo coroideo atípico y el gangliocitoma displásico del cerebelo, dadas las características parecidas a las de una masa de uvas en la región de la fosa posterior. El meduloblastoma se diferencia del gangliocitoma displásico por su mejor afinidad por el gadolinio. El ependimoma, el astrocitoma pilocitico y tumores teratoides-rabdoides atípicos se sitúan en ángulo cerebelopontino y ocupan las cisternas adyacentes, a diferencia del meduloblastoma cuya ubicación más frecuente es hacia la línea me$\operatorname{dia}^{1,5}$.

\section{Tratamiento}

El tratamiento de los meduloblastomas consiste en la resección quirúrgica seguido por radioterapia craneoespinal que trae consigo efectos colaterales significativos tales como déficit cognitivo y psicosocial mayor, disminución en la puntuación de coeficiente de intelectual y deficiencias endocrinológicas como hipotiroidismo e insuficiencia de la hormona del crecimiento ${ }^{5-8,15}$. Por ello, se ha comenzado a implementar la quimioterapia en altas dosis acompañada de la utilización de células madre como terapia de rescate en niños con un pronóstico completamente desfavorable 6-8,15.

Los estudios de seguimiento radioló- gico están elaborados para identificar recaídas tempranas, por lo que las terapias secundarias mejorarían de manera significativa el tratamiento. El seguimiento imagenológico debe ser considerado dentro de los esquemas de tratamiento debido a la alta tasa de recurrencia en sitios como fosa posterior, a nivel supratentorial y con menos frecuencia diseminación sistémica (huesos o ganglios linfáticos) que tienen los meduloblastomas ${ }^{5}$.

\section{Pronóstico}

Los niños con meduloblastoma en tratamiento con radiación craneoespinal y quimioterapia tienen un tiempo estimado de supervivencia alrededor de los 5 años en el $90 \%$ de los casos, además es importante resaltar que el pronóstico está bastante ligado a la edad siendo mejor en lactantes que en niños menores de 3 años en donde al parecer es moderado ${ }^{1,10}$. El grado de resección quirúrgica es importante para determinar la sobrevida de los pacientes, los pacientes con resección completa tienen mejor pronóstico. Los subtipos histológicos en conjunto con las características moleculares de los meduloblastomas se han implementado recientemente como agentes pronósticos de los mismos. Los tumores nodulares y desmoplásicos parecen tener un buen pronóstico, por otro lado, los tumores de células grandes/anaplásicos tienen un mal pronóstico ${ }^{4,5,7}$.

\section{Conclusiones}

El manejo neuroquirúrgico para el meduloblastoma asociado al apoyo neuroncológico es parte fundamental en el manejo de esta enfermedad. En las últimas décadas se ha evidenciado un aumento de la supervivencia de los pacientes. Un estricto seguimiento imagenológico permitirá detectar recurrencias. La individualización de los pacientes que padecen esta compleja patología permitirá un abordaje integral.

Recibido: 09 de enero de 2018 Aceptado: 15 de marzo de 2018 


\section{Referencias}

1. Tascu A, Rizea RE. A rare case of medulloblastoma with excessive nodularity : imagistic features. Rom Neurosurg 2014: 303-6.

2. Sousa R, Sá G, Reimão S, Lopes L, Ruivo J, Albuquerque L, et al. Adult cerebellar medulloblastoma: CT and MRI findings in eight cases. Arq Neuropsiquiatr 2003;19:199-203. https://doi.org/10.1590/S0004-282X2003000200008.

3. Velho V, Palande D, Kakani A, Mally R, Ghodgaonkar P, Jaiswal A. Bilateral Cerebellar Medulloblastomas: A Case Report And Review Of Literature. Internet J Neurosurg 2007; 5: 2-4.

4. Akyüz C, Varan A, Küpeli S, Akalan N, Söylemezoglu F, Zorlu F, et al. Medulloblastoma in children: A 32-year experience from a single institution. J Neurooncol 2008; 90: 99-103. https://doi.org/10.1007/s11060-008-9638-X.

5. Martínez León MI. Review and update about medulloblastoma in children. Radiol (English Ed 2011; 53: 134-45. https://doi.org/10.1016/ S2173-5107(11)70007-6.

6. Brandes AA, Paris MK. Review of the prognostic factors in medulloblastoma of children and adults. Crit Rev Oncol Hematol 2004; 50: 121-8. https://doi.org/10.1016/j.critrevonc.2003.08.005.

7. Emc M, Ayn SM, F D, Ec VD. Chemotherapy for children with medulloblastoma. Sophia 2010.

8. Padovani L, Sunyach MP, Perol D, Mercier C, Alapetite C, Haie-Meder C, et al. Common Strategy for Adult and Pediatric Medulloblastoma: A Multicenter Series of 253 Adults. Int J Radiat Oncol Biol Phys 2007; 68: 433-40. https://doi.org/10.1016/j.ijrobp.2006.12.030.

9. Bühren J, Christoph AH, Buslei R, Albrecht S, Wiestler OD, Pietsch T. Expression of the neurotrophin receptor p75NTR in medulloblastomas is correlated with distinct histological and clinical features: evidence for a medulloblastoma subtype derived from the external granule cell layer. J Neuropathol Exp Neurol 2000; 59: 229-40.

10. Borowska A, Jóźwiak J. Medulloblastoma: Molecular pathways and histopathological classification. Arch Med Sci 2016; $12: 659-66$. https://doi.org/10.5114/aoms.2016.59939.

11. de Haas T, Oussoren E, Grajkowska W, Perek-Polnik M, Popovic M, Zadravec-Zaletel L, et al. OTX1 and OTX2 Expression Correlates With the Clinicopathologic Classification of Medulloblastomas. J Neuropathol Exp Neurol 2006; 65: 176-86. https://doi.org/10.1097/01. jnen.0000199576.70923.8a.

12. Johnston DL, Keene D, Bartels U, Carret AS, Crooks B, Eisenstat DD, et al. Medulloblastoma in children under the age of three years: A retrospective Canadian review. J Neurooncol 2009; 94: 51-6. https://doi.org/10.1007/s11060-009-9799-2.

13. Sirachainan N, Nuchprayoon I, Thanarattanakorn P, Pakakasama S, Lusawat A, Visudibhan A, et al. Outcome of medulloblastoma in children treated with reduced-dose radiation therapy plus adjuvant chemotherapy. J Clin Neurosci 2011; 18: 515-9. https://doi.org/10.1016/j. jocn.2010.08.012.

14. Quinlan A, Rizzolo D. Understanding medulloblastoma. Am Acad Physician Assist 2017; 30: 30-6. https://doi.org/10.1097/01. JAA.0000524717.71084.50.

15. Kopač Š, Jereb B. Medulloblastoma in children 0-3 years old: Forty years' experience in Slovenia. Pediatr Hematol Oncol 2004; 21: 1721. https://doi.org/10.1080/08880010490263344.

\section{Correspondencia a:}

Dr. Luis Rafael Moscote-Salazar

Universidad de Cartagena, Cartagena de Indias, Colombia.

mineurocirujano@aol.com 